\title{
New Medical Therapies in Inflammatory Bowel Diseases In 2017
}

\author{
Atilla Ertan ${ }^{1^{*}}$ and Jamie Stewart ${ }^{2}$ \\ ${ }^{1}$ Ertan Digestive Disease Center - Inflammatory Bowel Diseases Center, USA \\ ${ }^{2}$ University of Texas Health McGovern Medical School (UTMMS) Gastroenterology, Hepatology and Nutrition Division and Memorial Hermann Hospital \\ - Texas Medical Center, USA
}

Received: August 11, 2017; Accepted: September 25, 2017; Published: October 09, 2017

*Corresponding author: Atilla Ertan, The UTMMS-MHH Ertan Digestive Disease Center and Gastroenterology Center of Excellence, 6400 Fannin, Suite 1400, Houston, Texas 77030, USA, Tel: 713-704-5928, 713-704-3450; Fax: 713-704-3485; E-mail: atilla.ertan@uth.tmc.edu

\section{Editorial}

A significant progress has been made regarding the management of inflammatory bowel disease (IBD) over the past 38 years as shown in Table 1. Our therapeutic goals in IBD are summarized in Table 2. Only limited treatment options existed until the introduction of anti-tumor necrosis factor (anti-TNF) agents, which was a landmark in the management of these debilitating diseases. It is recommended that patients with IBD be treated with a combination of anti-TNF agents with immunomodulator therapy to increase the likelihood of steroidfree induction and mucosal healing with long-term remission. According to our own experience and general consensus, the IBD treat-to-target strategy with anti-TNFs is well-respected to change the natural history of IBD with a deep remission in a significant number of IBD patients [1-2]. Early intervention with top-down therapy may be the future direction in selected moderate to severe IBD patients with close follow-up and trough level monitoring as needed. Selected conditions for the top-down treatment with anti-TNFs are shown in Table 3.

\begin{tabular}{ll}
\multicolumn{2}{l}{ Table 1: History of IBD Treatment } \\
Year & Drug \\
1979 & Sulfasalazine, Steroids \\
1980 & Antibiotics, Azathioprine, 6-MP \\
1993 & 5-ASA \\
1994 & Budesonide \\
1995 & Methotrexate \\
1998 & Infliximab \\
2007 & Second generation anti-TNF agents \\
2014 & New agents \\
$\mathbf{2 0 1 5}$ & Biosimilars \\
\hline
\end{tabular}

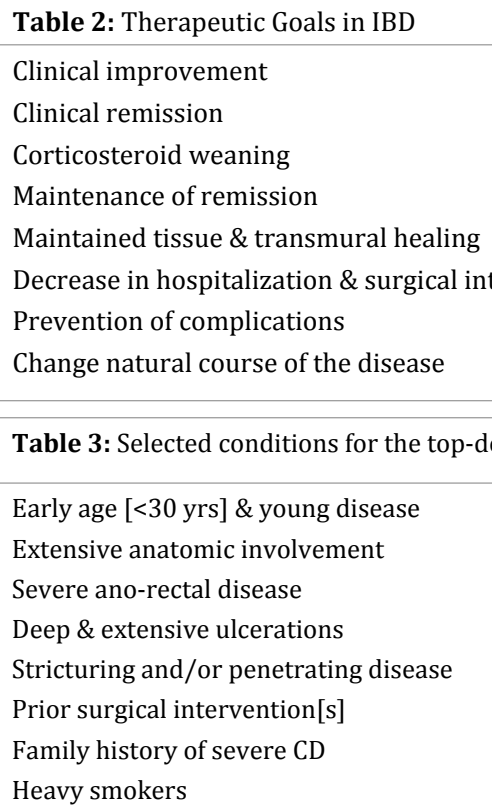

Early age $[<30$ yrs] \& young disease

Extensive anatomic involvement

Severe ano-rectal disease

Deep \& extensive ulcerations

Stricturing and/or penetrating disease

Prior surgical intervention[s]

Family history of severe CD

Heavy smokers

Table 3: Selected conditions for the top-down treatment with anti-TNFs

Therapies with anti-TNFs have reduced relapse rates and allowed mucosal healing and, as a result, improved long-term outcomes in a substantial proportion of patients. According to a large amount of studies, anti-TNFs induced approximately $30 \%$ clinical remission and 50\% clinical response in patients with moderate to severe Crohn's disease (CD) who are anti-TNF naïve or experienced. Moreover, the secondary loss of response may vary between 10 to $50 \%$ per year depending on studies and followup periods [3-6]. The "gut-selective" humanized, monoclonal antibody against alfa4beta7 integrin, [Vedolizumab] is now an established and FDA approved treatment option for patients with chronic ulcerative colitis (CUC) and CD either before or after anti-TNF therapy [7]. A recent study showed excellent rates of clinical remission at 12 months and mucosal healing, with no PML or other serious adverse events [8]. Recently, a fully human IgG1k monoclonal antibody that binds the p40 subunit of IL 
12/23 [Ustekinumab] was approved by the FDA for patients with moderate to severe $\mathrm{CD}$ [9]. In addition to initial very promising results with Ustekinumab, the phase III trials in patients with moderate to severe CD showed $34 \%$ clinical remission at week 6. Subsequent European studies with Ustekinumab revealed a relatively better clinical remission and continued to maintain clinical remission at 12 months [9-10]. Ustekinumab seems to be an attractive option with a relatively low immunogenicity, but its optimal dose has not been determined. All available biologic agents approved by the FDA are shown in Table 4.

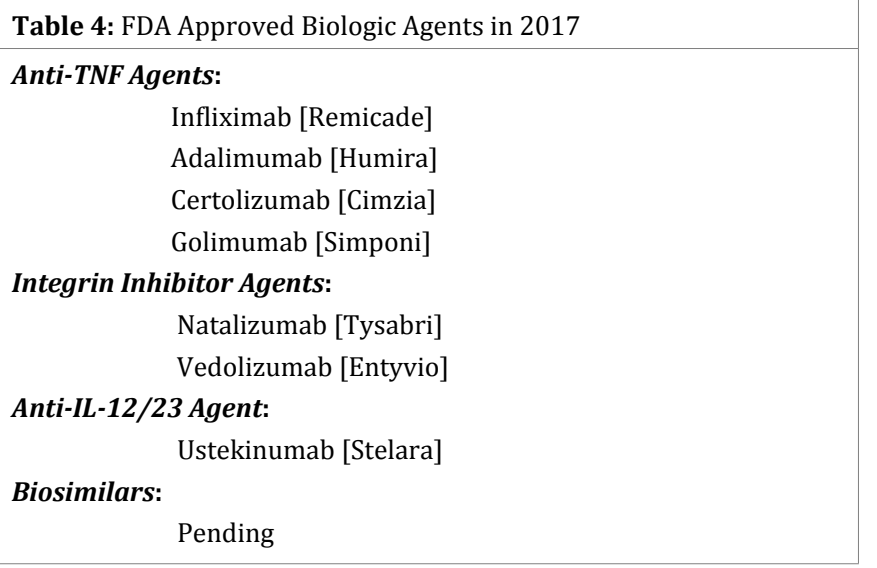

Despite the tremendous progress there are a significant number of patients who are refractory to these available biologic agents. Our understanding of the gut immune mechanism has become more sophisticated, but it still remains quite incomplete. The future looks more promising as several other novel treatment options have been identified and clinical studies are underway to determine the efficacy and safety of these therapies. Of note is the challenge of new clinical trials that have yielded positive earlyphase results, but do not translate into positive late-phase study outcomes due to heterogeneity of patients and disease type, high placebo rates and other factors. Having a number of different agents available will allow us to offer the best therapies for an individual patient. The majority of the novel agents in phase of development of the treatment of IBD are summarized in Table 5. There are novel ways of reducing inflammation by targeting downstream signaling such as Janus kinase inhibitors, Tofacitinib and Filgotinib; the target lymphocyte trafficking as new antiintegrin agent, AJM300 and sphingosine1P1R, Fingolimob; and antisense oligonucleotides to transforming growth factor-beta, Mongersen. It is important to note that all above mentioned small molecules are oral agents [11-18] and extensive clinical research is pending in our Center and around the world. These agents may have huge implications and potentially less costly. We have to wait to see their late phase effectiveness and safety studies within the next 5-10 years.

\begin{tabular}{|l|l|l|}
\hline \multicolumn{3}{|c|}{ Table 5: New Agents in Development } \\
\hline \multicolumn{1}{|c|}{ Name } & Major Effect & \multicolumn{1}{c|}{ Product } \\
\hline Etrolizumab & Anti-beta-7 & GI spec. integrin antagonist \\
Tocilizumab & Anti-IL-6 & Humanized MCA \\
Secukinumab & Anti-IL-17 & Humanized MCA \\
Risankizumab & Anti-IL-23 & Humanized MCA \\
Tofacitinib* & Anti-JAK & Immunomodulator \\
Fingolimod* & Sphingosine1P1R & Lymph. recept. agonist \\
Mongersen* & Targets SMAD7 & Antisense oligonucleotide \\
AJM300* & Anti-alfa-4 & Integrin antagonist \\
PPC* & Mucus & Phosphatidylcholine \\
*PO Agents & & \\
\hline
\end{tabular}

\section{Acknowledgement}

This study is supported by A. Ertan Research Education Foundation.

\section{Conflicts of Interest}

The authors are involved in clinical research with AbbVie, Janssen, Pfizer, Celgene, UCB, Takeda, F. Hoffman-LaRoche and Bristol-Myers Squibb. The research income stays in the Division for various other research and educational activities. There is no financial interest such as honoraria, participation in speakers' bureaus, membership, employment, consultancies, stock ownership, or other equity interests.

\section{References}

1. Colombel JF, Mahadevan U. Inflammatory bowel disease 2017: Innovations and changing paradigms. Gastroenterology. 2017;152(2):309-312.

2. Abraham C, Dulai PS, Vermerie S, Sandborn WJ. Lessons learned from trials targeting cytokine pathways in inflammatory bowel diseases. Gastroenterology. 2017;152(2):374-388. Doi: 10.1053/j. gastro.2016.10.018

3. Feagon BG, Lemann M, Befrits R, Connell W, D'Haens G, Ghosh S, et al . Recommendation for the treatment of Crohn's disease with tumor necrosis factor antagonists: an expert consensus report. Inflamm Bowel Dis. 2012;18(1):152-160. Doi: 10.1002/ibd.21870

4. Colombel JF, SanvornWJ, Reinisch W, Mantzaris GJ, Kornbluth A, Daniel Rachmilewitz, et al. Inflisimab, azathioprine, or combination therapy for Crohn's disease. N Engl J Med. 2010;362(15):1383-1395. DOI: 10.1056/NEJMoa0904492

5. Rutgeerts P, Van Assche G, Sanborn WJ, Wolf DC, Geboes K, Colombel JF, et al. Adalimumab induces and maintains mucosal healing in patients with Crohn's disease: data from the EXTEND trial. Gastroenterology. 2012;142(5):1102-1111. Doi: 10.1053/j. gastro.2012.01.035

6. Danese S, Colombel JF, Reinisch W, Rutgeerts P. Review article: infliximab for Crohn's disease treatment-shifting therapeutic strategies after 10 years of clinical experience. Aliment Pharmacol Ther. 2011;33(8):857-869. Doi: 10.1111/j.1365-2036.2011.04598.x 
7. Sandborn WJ, Feagan BG, Rutgeerts P, Hanauer S, Colombel JF, Bruce $\mathrm{ES}$, et al. Vedolizumab as induction and maintenance therpy for Crohn's disease. N Engl J Med. 2013;369(8):711-721. DOI: 10.1056/ NEJMoa1215739

8. Dulai PS, Singh S, Colombel JF, Peerani F, Narula N, Chaudrey K, et al. The real-world effectiveness and safety of Vedolizumab for moderate-severe Crohn's disease: Results from the US Victory Consortium. AJG. 2016;111(8):1147-1155. Doi: 10.1038/ ajg.2016.236

9. Sandborn WJ, Gasink C, Gao LL, Blank MA, Johanns J, Cynthia Guzzo, et al. Ustekinumab induction and maintenance therapy in refractory Crohn's disease. N Engl J Med, 2012;367:1519-1528. DOI: 10.1056/ NEJMoa1203572

10. Khanna R, Afif W. Ustekinumab for Crohn's disease. Gastroenterology. 2017;152(6):1616-1619.

11. Bouguen G, Chevaux JB, Peyrin BL. Recent advances in cytokines: therapeutic applications for inflammatory bowel diseases. World J Gastroenterol. 2011;17(5):547-556. Doi: 10.3748/wjg.v17.i5.547
12. Vuitton l, Koch S, Peyrin BL. Janus kinase inhibition with to facitinib: changing the face of inflammatory bowel disease treatment. Curr Drug Targets. 2013;14(12):1385-1391.

13. Perrier C, Rutgeerts P. New drug therapies on the horizon for IBD. Dig Dis. 2012;30(suppl 1):100-105. DOI:10.1159/000341133

14. Danese S. New therapies for inflammatory bowel disease: from the bench to the bedside. Gut. 2012;61(6):918-932. Doi: 10.1136/ gutjnl-2011-300904

15. Eietdjik ST, D'Haens GR. Recent developments in the treatment of inflammatory bowel disease. J Dig Dis. 2013;14(6):282-287. Doi: 10.1111/1751-2980.12048

16. Neurath MF. New targets for mucosal healing and therapy in inflammatory bowel disease. Mucosal Immunology. 2014;7(1):6-19. Doi: $10.1038 / \mathrm{mi} .2013 .73$

17. Dupont A, Ertan A. Medical therapy update in Crohn's disease. SOJ Immunol. 2014;2(1):1-2.

18. Neeraj N, Rubin DT, Sands BE. Novel therapies in inflammatory bowel diseases: An evaluation of the evidence. Am J Gastroenterol. 2016;3:38-44. Doi:10.1038/ajgsup.2016.19 